\title{
INVESTIGACIÓN/RESEARCH
}

Recibido: 16/08/2012---Revisado: 30/09/2012

Aceptado: 23/12/2012---Publicado: 15/03/2013

\section{LA ENSEÑANZA DE LA HISTORIA EN VENEZUELA: ALGUNAS RECOMENDACIONES}

María Elena Del Valle de Villalba': Universidad Metropolitana de Caracas. Venezuela. manedelvalle@gmail.com

\section{Resumen}

El presente artículo presenta las principales conclusiones a las que se llegó luego de realizar una investigación en la que se analizaron los principales atributos de la enseñanza de la Historia en Venezuela. Luego de analizar 3 textos escolares desde las técnicas del análisis del discurso, analizar el discurso docente de tres profesores de Historia de Venezuela y de describir la interacción generada en tres liceos de la capital en un aula de clase de la mencionada materia, se llegó a un conjunto de recomendaciones que acá se desarrollan.

Palabras clave: Discurso Pedagógico, Enseñanza de la Historia, Venezuela.

\footnotetext{
${ }^{1}$ María Elena Del Valle de Villalba, nacida en Caracas, Venezuela, el 10 de marzo de 1970, graduada de Profesora en la Universidad Pedagógica Libertador en el año 1992, con especialización en Historia Económica y Social de Venezuela en la Universidad Santa María, con Maestría en Historia Económica y Social de Venezuela, tesis con mención publicación y suma cum laude, Doctora en Ciencias de la Educación en el Pedagógico de Caracas (2010), Coordinadora del Núcleo de Investigaciones Geohistóricas Lisandro Alvarado, cuenta con proyectos financiados por la UPEL y con participación de la Universidad Complutense de Madrid, con proyectos financiados por el FONACIT, investigadora certificada de la Universidad Complutense de Madrid, el grupo internacional CONCILIUM y del CELARG, ponente regular en eventos nacionales e internacionales, adscrita a la línea de investigación, Ciencias del lenguaje y con siete años de investigación en el área de análisis del discurso. Profesora Ordinaria de la Universidad Pedagógica Libertador. Investigadora acreditada del CYTED, la AECID, profesora de la UNIMET con postdoctorado en semiótica y pragmática.
} 


\title{
THE TEACHING OF HISTORY IN VENEZUELA: SOME RECOMMENDATIONS
}

\begin{abstract}
This article presents the main conclusions reached after an investigation in which we analyzed the main attributes of the teaching of history in Venezuela. After analyzing three textbooks from the techniques of discourse analysis, discourse analysis of three professors teaching History of Venezuela and describe the interaction generated in three high schools in the capital in a classroom of that stuff, it was a set of recommendations that are developed here.
\end{abstract}

Key words: Speech Teaching, Teaching History, Venezuela.

\section{INTRODUCCIÓN}

Al analizar la enseñanza de la Historia en Venezuela se ponen en evidencia varios problemas. El primero de ellos está en relación con una denuncia frecuentemente efectuada por los profesores de Secundaria de todo el ámbito curricular: la falta de profundidad y pertinencia de la enseñanza de las Ciencias Sociales en General y de la Historia en particular puesta en evidencia en concreto en la poca sensibilidad en relación a los contenidos desarrollados, la débil importancia otorgada a su comprensión y lo desarticulado de su construcción en relación con las realidades nacionales. Ahora bien, dichas apreciaciones e intuiciones personales hay que comprobarlas de algún modo, de manera que se puedan documentar cuáles son las necesidades de los estudiantes, de los profesores y permitir así una adecuada actuación didáctica.

El segundo de los problemas, que puede contemplarse como causa-efecto del anterior, es la producción y aplicación de textos o manuales escolares con criterios explícitos que lejos de responder a los propósitos de la enseñanza de la Historia y a las competencias de los estudiantes que los usan son en muchos de los casos obstáculo para la comprensión de los contenidos desarrollados. Más allá de los aspectos más formales de la lengua, como pueden ser la sintaxis, el léxico o la ortografía que permitirían considerar satisfactorio un texto, en el caso de la enseñanza de la Historia se trata del abordaje de tópicos sensibles que derivan en la comprensión de abstractos de vital importancia, como el concepto de Historia, papel de los colectivos, valoración de los héroes, etc.

Luego de realizar la observación y análisis de las principales prácticas asociadas a la enseñanza de la Historia en Venezuela y a la vista de los aportes obtenidos al realizar esta investigación, expondremos a continuación las conclusiones más generales que se extraen de la misma. Para ello, fijaremos la atención en la manera en que se alcanzan los objetivos propuestos y respuesta a las preguntas de investigación, así 
como en las implicaciones de los aportes obtenidos. Para finalizar, señalaremos líneas futuras de investigación.

\section{METODOLOGÍA}

Consecución de los objetivos planteados y respuesta a las preguntas de investigación.

La presente investigación se planteó dar respuesta a una serie de preguntas de investigación derivadas de la pregunta motriz anunciada, a saber:

¿Qué atributos tiene el discurso utilizado por los docentes en la enseñanza de la Historia?

- ¿Qué características tiene el discurso no verbal usado por los docentes en la enseñanza de la Historia?

- ¿Qué implicaciones puede tener en la dinámica generada dentro del aula, el discurso usado por el docente en la enseñanza de la Historia?

- ¿Qué características tiene el discurso contenido en el los textos escolares usados para la enseñanza de la Historia?

- ¿Qué implicaciones puede tener la construcción discursiva del texto escolar en la formación de los estudiantes

- ¿Qué atributos posee la interacción generada dentro del aula de Historia entre el docente y el alumno?

- ¿Qué dinámicas discursivas se generan dentro del aula como consecuencia de la interacción entre: texto-alumno-docente?

- Interpretar el discurso pedagógico en la enseñanza de la Historia de Venezuela para construir un cuerpo de aportes teóricos.

Los objetivos que nos planteamos cumplir fueron:

- Interpretar el discurso pedagógico en la enseñanza de la Historia de Venezuela para construir un cuerpo de aportes teóricos.

- Establecer las características del discurso verbal y no verbal usado por los docentes de Historia de Venezuela.

- Analizar los atributos verbales y no verbales del discurso usado en los textos de Historia de Venezuela.

- Interpretar la interacción generada dentro del aula entre el docente y el alumno. 
- Comprender los elementos ideológicos presentes en los discursos del docente, el texto y en la interacción entre docente y alumno dentro del aula.

Al Llegar al final de esta investigación podemos ver con satisfacción que tanto los objetivos como las preguntas generadas a partir de los mismos se respondieron a lo largo del trabajo. Toda investigación exige la preocupación por la construcción de un conjunto ligeramente estable de ideas.

De la misma manera, debe considerarse que los aportes teóricos de investigaciones como esta, deberían generar la creación de ciertas tendencias que de alguna manera sirvan de contribución a investigaciones posteriores y del mismo modo orienten la actividad desarrollada en nuestra específica área de estudio.

Por otro lado, hace mucho rato que se están generando reflexiones que demandan el redimensionamiento de la enseñanza de la Historia en todos sus órdenes, de tal manera que esa conclusión no sería necesariamente "la tapa del frasco", es por esto que nuestras conclusiones pretenden no sólo apuntalar las preocupaciones antes descritas, sino también proponer humildemente una fórmula para su corrección.

\section{ANÁLISIS Y DISCUSIÓN}

Las implicaciones de los aportes teóricos obtenidos se desglosan en conclusiones y recomendaciones que se desarrollarán en este capítulo de la siguiente manera:

- Estás dando clases de Historia en Venezuela, en el siglo XXI: Ubícate.

- El texto es el pretexto: aprovéchalo.

- De la mesa de cuatro patas, los docentes son: Dos.

- De la mesa de cuatro patas, los alumnos son: Dos.

- Lo que estás enseñando es Historia, no otra cosa.

- La ideología: ADN ineludible

\subsection{Estás dando clases de Historia en Venezuela, en el siglo XXI: Ubícate.}

El contexto en una situación comunicativa es determinante. Dicho en forma breve, el contexto es el conjunto de características relevantes que rodean a una situación comunicativa. La cultura de quienes participan en la acción comunicativa, sus experiencias previas, sus estereotipos, sus prejuicios, su actitud, sus expectativas y necesidades e incluso, sus rasgos físicos son elementos fundamentales a tomar en cuenta al momento de establecer cualquier comunicación. Conocer el contexto es condición "sine quae non" para el buen entendimiento e interpretación y en nuestro caso concreto, lograr los objetivos de formación planteados. 
Regalar un vino exquisito a los que nos invitan a cenar en su casa es un gesto amable y decoroso, pero si quien nos convida es un ex-alcohólico rehabilitado, el gesto es grosero y hasta peligroso. El desconocimiento del contexto puede propiciarnos momentos desagradables, bochornosos y problemáticos. En este sentido esta anécdota ilustra un poco la situación que intentamos describir:

Asistí hace unos días a una boda en la que las dos familias

concurrentes apenas se conocían entre sí. En un momento de la ceremonia nupcial, una tía del novio que reside fuera de España, tuvo la cordialidad de levantarse para leer un pequeño escrito de su puño y letra, con mucho sentimiento, provocando la emoción de muchas de los presentes. En el climax de su oratoria y cuando las lágrimas eran

abundantes entre el público, la abuela de la novia gritó inesperadamente "Qué gorda es", "Qué gorda está"... Lógicamente, la reacción general fue la de una bochornosa vergüenza ajena. La abuela volvió a insistir, esta vez interrogando: "Pero ¿cómo está tan gorda?". La oradora, ante la grosera interrupción miró al público y con forzada sonrisa dijo, enjugando resignadamente las lágrimas: "Gracias". La abochornante abuela fue duramente reprendida por sus hijas en un

aparte, lejos del público extrañadísimo. Le gritaron muchísimo. Acercando sus bocas malhumoradas a las orejas de la mujer senil, que a duras penas conseguía entender qué había pasado o qué cosa mala había hecho. Y es que la buena de la abuela lleva casi diez años sin oirse a sí misma. Con un muy triste $98 \%$ de sordera. $Y$ sin audífono. Totalmente fuera de contexto. ${ }^{2}$

Un docente de cualquier cátedra, cuando la inicia debe tomar en cuenta todos los elementos que de una manera o de otra semantizan el lenguaje. No es lo mismo dar Historia que dar Química, No es lo mismo dar Historia en Venezuela, que darla en España. Conocer el contexto comunicacional es fundamental para el logro de los objetivos planteados. En el caso de España por ejemplo, la enseñanza de la Historia debería plantearse desde las especificidades de las comunidades autonómicas, con lo cual aunque se dicte Historia de España habrá que contextualizar aun más, ya que no es lo mismo dar Historia en la Comunidad autónoma de Madrid, que en Galicia. Si se viene de una dictadura, si ciertas epidermis han sido lastimadas, el lenguaje a utilizar, los ejemplos, las descripciones, todos esos elementos podrán hacer exitosa o terrible inútil la situación comunicativa.

En este sentido y refiriéndose específicamente a la enseñanza de la Historia en España afirma Almuiña (1998) que tanto por exigencias científicas (históricas) como para huir de planteamientos extracientíficos (instrumentalizadores), parece obligado ofrecer una visión general e integrada que sea respetuosa con el acontecer histórico. Evidentemente para alcanzar este objetivo posiblemente existan otros caminos, pero el recorrido que ha parecido mejor (completo, estructurado) y más sencillo para este

\footnotetext{
${ }^{2}$ Tomado de: http://cicuta.obolog.com/importancia-contexto-112764. Fecha de Consulta 22-08-2010
} 
tipo de alumnos ha sido seguir una evolución a través del hilo cronológico (sin que ello suponga ni mucho menos repetición de fechas y datos).

La historia como un continuum: partir de la prehistoria para llegar al tiempo presente en el cual el alumno tiene la necesidad de ubicarse a partir del conocimiento de unas raíces y la génesis de buena parte de sus problemas y operatividades p 45. Esta propuesta de ver la Historia de España como un continuum contextualizado, donde cada comunidad autónoma responde a una Historia nacional y a la vez a las especificidades de las comunidades autonómicas es la visión que conllevará a una Historia contextualizada.

El contexto histórico en el caso de Venezuela es vital. Ya la polarización que se experimenta fue descrita en líneas previas con lo cual no redundaremos en ello, lo que sí es determinante es que todos los factores que de alguna manera semantizan la información sean tomados en cuenta. El alumno al que le enseñamos y sus atributos son otro elemento crucial. No son niños, en su mayoría sus edades están comprendidas entre los 17 y 19 años.

Las características de los jóvenes en esas edades son:

- Hay una autoimagen inestable, generalmente sintiéndose poco valiosos y no contentos con como son.

- Es una época de importantes decisiones: elección de vocación y de pareja.

- Están en un proceso complejo como se ha descrito en líneas previas, conocer a tu audiencia es otro elemento fundamental. Conocer los problemas a los cuales se enfrentan, establecer un nexo que les haga saber que les conoces es clave.

- Características sociales:

- Hay una intensa relación con el grupo de pares.

- Revisión crítica de los valores

- Se comienza a desarrollar el sentido de justicia.

- Discusión de derechos y deberes.

Estas son edades en las que podemos ejercer gran influencia, momentos determinantes en los cuales el joven puede descubrirse capaz de escribir su futuro por lo que un discurso cercano sin dejar de ser contundente puede ser una estrategia efectiva.

Dependiendo de la extracción social de tu alumno el discurso deberá adaptarse, no le deberás hablar usando el mismo lenguaje ni los mismos argumentos a uno del estrato I que a uno del estrato IV. Los problemas que enfrentan son distintos y si el discurso no discrimina estas diferencias su efectividad podrá verse vulnerada. Nuestro país, como muchos de Latinoamérica se enfrentan a altas cifras de deserción escolar, 
muchas veces motivada esta por un discurso elitesco y desarticulado en el aula que no les motiva.

El informe de la PREAL (2005) señala que:

En las zonas urbanas, las niñas abandonan la escuela con menor frecuencia que los niños; progresan a lo largo del ciclo escolar con menor repetición y una mayor proporción de las jóvenes que de los varones de entre 15 y 19 años de edad egresan de la educación secundaria sin retraso. En las zonas rurales, en cambio, las mujeres tienden a dejar la escuela más tempranamente que los hombres, particularmente durante los primeros años de la primaria, y en algunos países (Bolivia, Guatemala, Paraguay, Perú y República Dominicana) un porcentaje mayor de niñas que de niños no ingresan al sistema o lo abandonan sin completar el primer grado p 54.

Lo anterior introduce una variable adicional que es la ubicación geográfica. Si viven en un barrio ${ }^{3}$ o en una urbanización, si se transportan en metro o tienen vehículo propio son elementos que pueden permitirnos hacer un "gancho" que haga que este adolescente se sienta tomado en cuenta e involucrado en su proceso de formación.

En Chile, Colombia, México, Panamá, Perú, Uruguay y Venezuela entre $50 \%$ y $60 \%$ del abandono escolar se produce en el transcurso de la secundaria y, con excepción de Chile, en todos ellos la deserción se concentra más en el comienzo que en el final del ciclo, de lo cual podríamos inferir que en un país con graves problemas económicos como el nuestro el alumno (a) prefiere muchas veces ir a trabajar, que permanecer en un entorno en apariencia inútil y sin sentido.

El mencionado informe señala como principales causas de la deserción las siguientes:

Seis tipos de razones que explican la deserción:

- PREAL (2005) Razones económicas, que incluyen tanto la falta de recursos del hogar para enfrentar los gastos que demanda la asistencia a la escuela, como el abandono para trabajar o buscar empleo.

- Problemas relacionados con la oferta o falta de establecimientos, que reúne como motivos la inexistencia de escuela o, dentro de ella, de nivel o grado, su lejanía y las dificultades de acceso, además de la ausencia de maestros.

\footnotetext{
${ }^{3}$ En Venezuela y República Dominicana se denomina barrio a las zonas deprimidas de las ciudades, caracterizadas por la construcción de viviendas precarias y la carencia parcial o total de servicios básicos; corresponde a lo que en que en otros países denominan: favela (Brasil); barrio de chabolas (España); villa miseria (Argentina); colonia popular o colonia proletaria (México); cantegril (Uruguay); población (Chile); pueblo joven (Perú); invasión (Colombia) y (Ecuador); ciudadela (Bolivia).
} 
- Problemas familiares, que comprende las razones más frecuentemente mencionadas por las niñas y las adolescentes: la realización de quehaceres del hogar, el embarazo y la maternidad.

- Falta de interés, que incluye la falta de interés de los padres.

- Problemas de desempeño escolar: bajo rendimiento, problemas de conducta y problemas asociados a la edad.

- Otras razones: discapacidad, servicio militar, enfermedad o accidente, asistencia a cursos especiales, y otras $p 78$.

Entre las razones inmediatas que aducen los jóvenes para la deserción escolar, más del $70 \%$ de ellos señala haberse retirado por dificultades económicas o porque se encuentran trabajando o buscando un empleo. De esta manera, si los problemas con los que lidia el joven que está en el aula, es la manera de colaborar con los retos económicos de su hogar, de poco le valdrá un discurso elitesco, memorístico, caletrero de hechos que se han quedado en el pasado. Para concluir este apartado, Ubicarse en los contextos: geográfico, social, psicológico, histórico es fundamental para realizar intercambios aúlicos apropiados.

\subsection{El texto es el pretexto.}

Al acercarnos al final de esta investigación y luego de haber observado e interpretado las características y el uso del libro de texto en la enseñanza de la Historia, pudimos generar una suerte de clasificación de los textos que a continuación se describe.

Del corpus de 3 textos que analizamos así como de otros que pudimos revisar en el desarrollo de la investigación surgió la siguiente clasificación:

\subsubsection{Texto-muleta}

El texto escolar que de manera bancaria suministra toda la información de manera acabada, que no reta las capacidades intelectuales del alumno-lector, que presenta los contenidos conceptuales de manera rígida y sin ningún dinamismo es lo que denominamos un texto-muleta.

A decir de Fontaine e Eyzaguirre (1997) los textos de estudio no sólo "transportan" el currículum sino también, en gran medida, lo determinan. Esto es especialmente cierto en los casos, como el nuestro, en que el currículum oficial está expresado como objetivos muy amplios o generales. Corresponde entonces a los textos ( $y$ también por cierto a los profesores) especificar y decidir por qué medios lograr estos objetivos, qué alcance y volumen tendrán los contenidos y también qué nivel de dificultad se exigirá. De esta manera, si los contenidos, así como las actividades sugeridas como comprobación de los mismos no sacan de la zona de confort al estudiante sino que por el contrario le sirven de apoyo hasta el punto de que adormecen cualquier iniciativa. 


\subsubsection{Texto-catecismo}

Con este calificativo describimos aquel libro de texto cargado de afirmaciones teñidas de ideología que asemejan a los catecismos religiosos de otrora. Las oraciones parecen balas que definen la Historia desde y para una ideología determinada, quedando asentadas de manera incuestionable. Las actividades que se colocan como parte de la comprobación de los aprendizajes son más una recapitulación o repetición de lo antes explicitado que la generación de procesos mentales complejos. Un texto de calidad puede ser un texto-catecismo, sus planteamientos pueden ser con base teórica y científica inobjetable pero, nuestra de idea de enseñanza de la Historia va más allá.

Cuando nos referimos a la calidad de una cosa, en este caso del libro de texto, queremos expresar también "algo más", algo que va más allá de su efectividad, de su relevancia y su equidad y que tiene que ver con lo que podríamos llamar "excelencia" y también "inspiración". En educación podría ser "esa cualidad extra de inventiva, estimulación, entusiasmo... que se encuentra, aunque rara vez, en algunos colegios y en algunos maestros" H. Hawes y D. Stephens (1990) esas cualidades deberían estar en el libro de texto, es decir, retar tanto al docente como al alumno (a) a generar ideas nuevas, incluso contrarias a las expuestas en él.

\subsubsection{Texto-Placebo}

El efecto placebo es definido como:

(...) como una intervención diseñada para simular una terapia médica, la cual no tiene un efecto específico para la condición que está siendo aplicada. Vale decir, corresponde a un procedimiento o intervención que no tiene un efecto fisiológico ni bioquímico sobre la enfermedad o condición en estudio. Si bien el placebo es una sustancia o intervención en teoría inerte y sin efectos sobre el curso de la condición en estudio, puede mostrar un perfil de efectos secundarios similares al fármaco estudiado ( $p$ 98).

Un libro de texto sin efecto, completamente nulo en la formación del alumno. Un conjunto de texto que si bien es verdad, abordan los contenidos requeridos por los programas, no tienen efecto alguno en el estudiante ni en el docente que de él hace uso. Cierto es que el centro de la dinámica aúlica no es el texto, pero sin duda un texto bien estructurado, con actividades originales, interesantes, actuales, contextualizadas puede ayudar en la generación de dinámicas discursivas importantes.

\subsubsection{Texto- Ladrillo}

Con este calificativo algo duro, si a ver vamos calificaron algunos estudiantes con los que se conversó a lo largo de la investigación a un texto, impenetrable, elitesco, inaccesible, bordado de terminología técnica y científica pero al final del día: duro como un ladrillo. 
Todo autor, al momento de escribir debe considerar como ya se señaló, las características del posible lector. En el caso de un libro de texto, conocemos sus características generales, sabemos que competencias de manera elemental debe manejar, con lo cual es un poco torpe presentarles un texto que parece escrito para miembros de una academia o para la ponencia de algún congreso.

Zuev (1998) señala que al libro de texto le corresponden las siguientes funciones didácticas:

- Informativa. Presentación de la totalidad de la información indicada por el programa de la respectiva asignatura.

- Transformadora: En dos sentidos: 1) reelaboración didáctica de los contenidos; 2) conversión de la actividad puramente cognoscitiva de los estudiantes en actividad transformadora.

- Sistematizadora: Exposición del material docente en una secuencia rigurosa sistematizada, para que el estudiante domine los procedimientos de la sistematización científica.

- De consolidación y de control. Contribución para que los estudiantes se orienten en el conocimiento adquirido y se apoyen en él para realizar la actividad práctica.

- De auto- preparación. Formación en los estudiantes del deseo de aprender y de la capacidad de aprender por sí mismos.

- Integradora. Ayuda a los estudiantes para asimilar y seleccionar los conocimientos como un todo único.

- Coordinadora. Aseguramiento del empleo más efectivo y funcional de todos los medios de enseñanza y del uso de medios extra- docentes de información masiva.

- Desarrolladora y educadora. Contribución a la formación activa de los rasgos esenciales de la personalidad armónica y desarrollada ( $p 45$ ).

Por lo tanto si de se queda solo en la Informativa, por muy valiosa que esta información sea, al no llegarle al estudiante ¿qué sentido puede tener?

\subsection{De la mesa de cuatro patas, los docentes son: Dos.}

Martínez-Otero (2007) afirma que: "El discurso docente es praxis facilitadora del desarrollo de la personalidad, ya que por medio del discurso el profesor proyecta su propia visión del mundo y orienta la trayectoria vital de los alumnos. El lenguaje docente puede promover el desarrollo intelectual, emocional, moral y social del educando, según las metas que persiga. El empleo diferencial del discurso en el aula, 
es consecuencia de la particular cosmovisión del docente, da lugar a diversas modalidades de relación profesor-alumno y genera variaciones significativas en la educación, pues se enfatizan ciertas dimensiones en perjuicio de otras" (p 45).

De lo anteriormente descrito se desprende que el papel del docente es fundamental, es lo que mantiene a la mesa en pie, le da equilibrio y sentido a la comunicación aúlica. Luego de largos meses de observación generamos una clasificación de docentes de acuerdo a sus atributos en el uso del discurso, la cual describimos a continuación:

\subsubsection{Docente-catedrático}

Esta tipología de docente se nos presenta como un académico preparado que despliega frente a sus estudiantes un discurso bien estructurado y con inobjetable base teórica. Su preocupación es fundamentalmente ofrecer informaciones a sus alumnos. Sus clases son eminentemente magistrales, casi monólogos en los cuales el alumnado no tiene participación alguna. Es un tipo de docente tradicional y algo ortodoxo que asume todo el protagonismo y que no favorece la interacción en el aula, de hecho podría decirse que, provocada por él no se genera ninguna. Es un técnico, un catedrático, un académico que aun cuando observa y cumple la trasmisión de los objetivos no maneja elementos de motivación ni busca ningún tipo de vinculación con los estudiantes, transmite contenidos a los escolares, pero se olvida de la dimensión humana.

\subsubsection{Docente-"pana"4}

Esta es una tipología algo curiosa, se trata de un docente que busca como propósito fundamental la familiaridad con el estudiantado, copia su lenguaje e intenta conversar sobre sus intereses personales, lo cual no sería problema si a la vez no se perdieran de vista los objetivos que les reunieron en el aula en primer término. En esta tipología predomina la vertiente emocional del discurso. Es el tipo de profesor que se interesa por los problemas y el desarrollo afectivo de sus alumnos, pero descuida los aspectos técnicos de la educación. Se desatiende la formación intelectual del alumno. Naturalmente un ambiente presidido por la cordialidad es necesario, pero sin que la educación se agote en el cultivo de la vertiente afectiva. Las horas de clases no deben pasarse en un concierto de anécdotas especulativas y opináticas que si bien generan lazos entre el docente y el alumno, descuidan el logro de los objetivos de la cátedra.

\subsubsection{Docente-candidato}

Esta tipología describe al docente que busca ante todo atraer a sus alumnos. Su uso del discurso es casi histriónico, sube y baja la voz, casi actuando los contenidos. En casos extremos hallamos un docente con un discurso hueco. Hay gran preocupación por la imagen, pero no se promueve la formación de los educandos, únicamente se los

\footnotetext{
${ }^{4}$ Pana" en Venezuela es compañero, amigo, pero uno que sea allegado. Ej: Luis es mi pana También se refieres a cuando alguien es amigable, simpático, llevadero. Ej: conoces a Carla? ella es muy pana. No tiene distinciones de sexo; ella y el pueden ser panas.
} 
entretiene, se les emociona. La influencia de la realidad venezolana ya descrita en líneas anteriores y de los medios masivos de comunicación conducen cada vez más a los docentes a adoptar modos de enseñar análogos a los presentadores de programas de televisión o de opinión.

En algunos casos su posición política es tan obvia y avasallante que los alumnos se pliegan a ella con el objeto de no ser el centro de atención o por miedo a posibles sanciones.

\subsubsection{Docente-profeta}

Es el típico maestro regañón, sus clases son eternas letanías en las que desde la sabiduría de la experiencia les habla a los alumnos para sacarlos de la oscuridad. Sermonea a los escolares. A menudo reprende a los alumnos por su comportamiento dentro y fuera del aula. Trata de reformar las "malas costumbres" de los educandos por medio de moralina. Como sus enseñanzas son inoportunas y superficiales no forma a sus alumnos, aunque es posible que sigan su "código de conducta" por temor a los castigos. Sus clases carecen de interacción y los alumnos entran en "modo ahorrador de energía" es decir, se desconectan cayendo en un mutismo total.

Ahora bien, ¿Cómo debería manejarse un docente dentro del aula para ser un instrumento adecuado? ¿Pueden en una sola persona estar todos estos atributos? ¿Son nocivos por separado? ¿Podrían incluirse todos en la dinámica aúlica? Nos parece que el docente ideal sería aquel que tuviera del catedrático la solidez teórica y de conocimiento, del "pana" la cercanía y el conocimiento de los alumnos, del candidato la capacidad para capturar la atención e involucrar a los estudiantes y del profeta la disposición a enseñar y compartir desde su experiencia.

\subsection{De la mesa de cuatro patas, los alumnos son: Dos.}

El alumno como bien lo afirma el sub-título el alumno es la clave junto con el docente del éxito dentro del aula. El docente puede ser la mezcla mágica del: académico, el pana, el candidato y el sacerdote y aun así no generar los resultados esperados. El alumno tiene que involucrarse, tiene que estar dispuesto y sentirse incluido en la dinámica aúlica.

De la misma manera en la que la investigación generó una tipología de docentes, del mismo modo y por reacción se produjo también una tipología de alumnos en función de lo observado. Dicha clasificación se describe a continuación:

\subsubsection{Alumno-secretario}

Alumno-secretario: está sometido a monólogos insufribles del "profesor- catedrático", sus lecciones son eternas, y como buen secretario toma nota de cada una de sus palabras las cuales deberá repetir de manera textual si quiere aprobar, víctima de un discurso dogmático y de un proceso de enseñanza memorista. Es el escolar que, en un marco en el que se le da preponderancia a la reproducción de contenidos, repite la 
lección sin reflexión ni comprensión. Este tipo de alumno es un mero receptor que almacena o colecciona informaciones ajenas. La relación es completamente bancaria tal como es definida por Freire (1992)

\begin{abstract}
En la visión «bancaria» de la educación, el «saber», el conocimiento, es una donación de aquellos que se juzgan sabios a los que juzgan ignorantes. Donación que se basa en una de las manifestaciones instrumentales de la ideología de la opresión: la absolutización de la ignorancia, que constituye lo que llamamos alienación de la ignorancia, según la cual esta se encuentra siempre en el otro ( $p$ 77)
\end{abstract}

Con frecuencia ambos, docente y alumno son siervos del libro de texto, texto-muleta, herramienta que no hace sino ocultar la falta de iniciativa del profesor para utilizar otros recursos complementarios y más enriquecedores.

\title{
3.4.2. Alumno-utilitario
}

Esta tipología de alumno se nos presenta como un elemento casi parasitario del docente-pana, espera en cada situación, que su amigo profesor sepa entender sus deficiencias y le suministre las soluciones que requiere. Es el alumno mimado, muy dependiente del profesor que alude siempre a argumentos emotivos para evadir sus responsabilidades. Esta enseñanza puede generar desvalimiento y subordinación emocional del alumno respecto del profesor, hasta el punto de que se impide o frena su desarrollo armónico y saludable. La afectividad ha de cultivarse, en todos los niveles, su importancia no se discute pero esto debe hacerse sin que se llegue a soslayar las demás vertientes de la educación.

\subsubsection{Alumno-prosélito}

Su condición es delicada, como consecuencia del discurso eminentemente persuasivo está muchas veces a merced de los argumentos manejados por el docente. Es el que germina en las aulas donde mora el "docente-candidato". Emerge así la tipología de alumno-prosélito que busca ser aceptado y que sigue a su docente por temor a la sanción. Si el alumno posee un cierto grado de desarrollo y un juicio crítico más o menos formado se protege de los sermones con una saludable actitud de rebeldía. En cambio, la resistencia es escasa o nula en el caso de los alumnos inseguros, inestables emocionalmente y con baja autoestima.

\subsubsection{Alumno-ausente}

Esta tipología de alumno es una de las más comúnmente observadas en las aulas de clases. Tal vez como consecuencia de los discursos antes descritos, o por la nociva combinación de todos ellos los alumnos se desvinculan, sacan hojas y garabatean en ellas. En el mejor de los escenarios sacan los nuevos "mejores amigos" de nuestros estudiantes, los celulares, chatean intercambiando informaciones realmente 
importantes con sus coetáneos. Otros más delicados, te miran con una suerte de expresión perdida, sus ojos están fijos en ti, pero su mente está en otro lugar.

\subsection{Lo que estás enseñando es Historia, no otra cosa.}

La escuela historiográfica desde la que creemos debe partirse al momento de enseñar la Historia como asignatura es la denominada Escuela de los Annales. Las características de esta fueron ya descritas en las bases teóricas de nuestra investigación y en este último apartado nos limitaremos a recapitular algunas definiciones de Medina Rubio (2002) que sirvan de orientación metodológica para los docentes.

La Historia desde esta perspectiva parte de las siguientes bases:

1) "Historia" remite a los acontecimientos vividos por los grupos humanos en el tiempo ${ }^{5}$, en este sentido, abarca un sinnúmero de sucesos de la vida, desde el pasado más remoto hasta nuestro tiempo actual. Vista así, la historia es el pasado, presente y futuro posible de la actividad humana, "un fenómeno ilimitado" del ser social.

2) la historia que constituye el día a día de la gente en su lenguaje y en su actuar, en este sentido es el conjunto de huellas cotidianas que deja el hombre en su paso por el tiempo y que son recordadas, las representaciones de la memoria colectiva de las comunidades, memoria de un valorado pasado que forma parte de los pueblos y que moldea su identidad, la cual se materializa sea de forma escrita o verbal en los mitos y leyendas y, en otros tiempos, las anécdotas, los cantos y los poemas, como bien pueden conocerse de la memoria de nuestros pueblos ancestrales y en las crónicas costumbristas de los periódicos o en las memorias que algún particular escribió en textos para la posteridad ( $p 89$ ) .

De esta manera, no es un contenido cualquiera, es una ciencia social que amerita un modo de enseñanza especial. Cuesta et al (2009) señalan una serie de tareas que consideramos de importancia si se quiere entender la complejidad de lo que enseñamos, las mismas son:

- Poner el presente y sus problemas en primer lugar, siguiendo la huella del genealogista, pero también de la sociología histórica y de algunos cultivadores de la historia del presente. Sólo en ese sentido, y sólo en ése, se puede admitir que la "historia cultural es historia del presente" (Popkewitz, Franklin y Pereyra, 2003).

- Entender la historia del presente como socio-génesis, como cambiantes y sucesivas construcciones socio-históricas de tradiciones inventadas.

\footnotetext{
${ }^{5}$ Nótese la aplicación del término "tiempo" para deslindarse de la visión centrada en el pasado, característico de las escuelas positivistas y romántica tradicional.
} 
- Comprender la socio-génesis de manera no finalista, no determinista y no continuista, dejando espacio para las configuraciones críticas del pasado, del presente y del futuro.

- Multiplicar y combinar las duraciones temporales. Hacer una historia efectiva, esto es, paradójica y contradictoria respecto a los usos y escalas de los tiempos y de los objeto de observación.

- Pero, a pesar de lo anterior, practicar una historia inteligible en su evolución temporal, marcando las continuidades, los cambios, las diversas direcciones, las tendencias predominantes y el significado histórico de los fenómenos estudiados.

- Superponer la mirada macroscópica con la profundización micro-histórica a través de biografías profesionales y estudios de caso ( $p$ 67).

Se trata entonces de un abordaje transdisciplinario en el que la Historia es un contenido vivo, actual, útil, comprensible que da sentido y explica las realidades actuales, es desde esta perspectiva que la Historia debe enseñarse.

\subsection{La Ideología: ADN ineludible}

El teórico fundamental desde el cual definimos ideología es Foucault (1993). El mencionado autor genera dos definiciones: una de carácter positiva, en la que se identifica al concepto con una práctica discursiva circunscrita en un ámbito del saber y de la sociedad, que es ajeno al interés de su quehacer arqueológico y genealógico y otra consideración que está marcada por un fuerte desdén y se refiere a la ideología como noción eterna inserta en una concepción de la historia fundada en totalizaciones y continuidades.

En principio, Foucault (1994) define a la ideología en su segunda connotación como un ídolo y un prejuicio, y se da a la tarea de destruirla poniendo en práctica la metáfora nietzscheana de filosofar con el martillo. De aquí deriva su método, definido como el dispositivo Foucault, que concibe a la teoría como caja de herramientas. Su objetivo es proponer instrumentos de descripción y análisis en vez de sistemas, enmarcarlos en una reflexión histórica sobre situaciones dadas.

Sea interpretada de la primera o de la segunda manera, la ideología es inevitable, no hay discurso que no la posea, ni conocimiento que no pueda verse influenciado por ella, de allí su comparación con el ADN.

Cualquier contenido es susceptible a sus efectos pero lo es más aun, el contenido histórico en un contexto altamente polarizado como el actual. ¿Cómo debe ser entonces la interacción aúlica?, ¿Debe vetarse la ideología? O lo que es más complejo, ¿Puede dejársele fuera del aula? 


\section{CONCLUSIONES}

Luego de realizar esta investigación, hemos llegado a las siguientes conclusiones:

- La presencia de la ideología no solo es inevitable, sino necesaria. El alumno debe estar expuesto a ella pero en la libertad de disentir de ella. El aula no puede convertirse en un partido político, ni en un mitin, debe ser un lugar de debate y discusión donde el alumno vaya formando su "caja de herramientas" en libertad e independencia.

- La Historia no puede verse desde un solo lado, son muchos los actores, muchas las historias y muchos los silencios que deben encontrar en el aula un lugar de respeto y tolerancia.

- La clase de Historia no es una prueba de "pulso" en el que alguno tiene que ganar. Si al concluirla no hay conclusión definitiva, esa será la mejor excusa para continuar la próxima.

\section{BIBLIOGRAFÍA}

Almuiña, Carlos (1998) Humanidades en la Historia de España en la ESO. Revista Ayer. Nro 30. Universidad de Valladolid. España.

Crow $\mathrm{R}$, Gage $\mathrm{H}$, Hampson $\mathrm{S}$, et al . The role of expectancies in the placebo effect and their use in the delivery of health care: a systematic review. Health Technology Assessment Vol.3: No.3. 1999. pp 96. The National Coordinating Centre for Health Technology Assessment (NCCHTA).

Cuesta et al (2009) Modos de educación y problemas de periodización histórica desde una perspectiva genealógicaseminario sobre transiciones, cambios y modos de educación centro internacional de la cultura escolar (CEINCE) Berlanga de Duero, 2 y 3 de julio de 2009. Fedicaria.

Fontaine y Eyzaguirre (1997) Por qué es importante el texto escolar. Estudios Públicos. Nro.68. Centro de Estudios Públicos. Kansas. Estados Unidos.

Foucault, M. (1993). Las redes del poder. Buenos Aires: Almagesto.

Foucault, M. (1994). El orden del discurso. Buenos Aires: Tusquets.

Freire, P. 1992. Pedagogía del oprimido. Madrid: Siglo XXI

H. Hawes y D. Stephens, Questions of Quality, Primary Education and Development (Essex: Longman, 1990).

Machado, A. (1936). Juan de Mairena. Madrid: Espasa-Calpe, 21996, Cátedra. 
Martinez-Otero, Valentín (2007) Modelo pedagógico del discurso educativo y su proyección en la calidad docente, discente e institucional. Revista Iberoamericana de Educación, núm. 2 Vol. 43 (enero-abril 2007), páginas 30-35

Medina Rubio, A. (2002). Lecturas de la Historia Regional y Local. Caracas: Casa Editorial de las Letras Andrés Bello.

Popkewitz, T., Franklin, B. M. y PEREYRA, M. A. (2003), Historia cultural y educación. Ensayos críticos sobre conocimiento y escolarización. Barcelona, Pomares.

PREAL. (2005) Deserción escolar un problema urgente. Formas y Reformas de la Educación. Diálogo Interamericano. Año 5. Nro. 14. Santiago de Chile. Chile.

\section{María Elena Del Valle de Villalba}

Nacida en Caracas, Venezuela, graduada de Profesora en la Universidad Pedagógica Libertador en el año 1992, con especialización en Historia Económica y Social de Venezuela en la Universidad Santa María, con Maestría en Historia Económica y Social de Venezuela, tesis con mención publicación y suma cum laude, Doctora en Ciencias de la Educación en el Pedagógico de Caracas (2010). 